Math. Model. Nat. Phenom.

Vol. 6, No. 2, 2011, pp. 160-172

DOI: $10.1051 / \mathrm{mmnp} / 20116206$

\title{
Transport Equation Reduction for a Mathematical Model in Plant Growth
}

\author{
S. Boujena ${ }^{*}$, A. Chiboub ${ }^{1}$ and J. Pousin ${ }^{2}$ \\ ${ }^{1}$ Department of Mathematics and computing, \\ Hassan II University, Sciences Faculty, \\ POB 5366 Maarif, Casablanca, Morocco \\ ${ }^{2}$ Université de Lyon, \\ INSA de Lyon, ICJ UMR CNRS 5028, 69100 Villeurbanne cedex France
}

\begin{abstract}
In this article a variational reduction method, how to handle the case of heterogenous domains for the Transport equation, is presented. This method allows to get rid of the restrictions on the size of time steps due to the thin parts of the domain. In the thin part of the domain, only a differential problem, with respect to the space variable, is to be approximated numerically. Numerical results are presented with a simple example. The variational reduction method can be extended to thin domains multi-branching in 3 dimensions, which is a work in progress.
\end{abstract}

Key words: plant growth, nutrients, hormones, concentration, transport, domain decomposition, asymptotic expansion, MAPDD

AMS subject classification: 35Q92, 92C80

\section{Introduction}

Plant growth presents a seasonal periodicity. The narrow exterior plants part where cells proliferate is called Apex. It's considered as a surface and its displacement represents the plant growth. The nutrients, produced in the roots and transported through the whole plant, are essential to its growth. The branches appear and grow according to some specified rules. Moreover the emergence of new buds is consequence of the localized concentration of many hormones, especially Cytokinin and Auxin. We assume that cells conducting the nutrients and hormones are in liquid solution. Auxin is produced in the Apex and Cytokinin in both the roots and the growing plant parts. The dynamics

*Corresponding author. E-mail: boujena@yahoo.fr 
of main branch growth and formation of new buds is modelised in [4]. The one-dimensional mathematical model is justified if the length (or height) of the plant, denoted by L, is essentially greater than the diameter of its trunk [4]. The growth speed $u(t)=L^{\prime}(t)$ depends on nutrient concentration $C$ and on the GM-factor concentration $R(t)$ at $x=L(t)$.

The concentrations of nutrient $\mathrm{C}$, and of hormones Auxine A and Cytokinin $\mathrm{K}$ are described by the advection-convection-diffusion equations with convective terms [4]:

$$
\begin{aligned}
& \frac{\partial C}{\partial t}+u \frac{\partial C}{\partial x}=d \frac{\partial^{2} C}{\partial x^{2}} \\
& \frac{\partial K}{\partial t}+u_{K} \frac{\partial K}{\partial x}=d_{K} \frac{\partial^{2} K}{\partial x^{2}}-\mu_{K} \\
& \frac{\partial A}{\partial t}+u_{A} \frac{\partial A}{\partial x}=d_{A} \frac{\partial^{2} A}{\partial x^{2}}-\mu_{A} \\
& x=0 ; L=L_{0} ; R=R_{0} ; C=C_{0} ; K=K_{0} ; \quad \frac{\partial A}{\partial x}-\gamma A=0 \\
& x=L(t) ; d \frac{\partial C}{\partial x}=-g(R) C ; d \frac{\partial K}{\partial x}=\alpha u ; d \frac{\partial A}{\partial x}=\alpha u
\end{aligned}
$$

where $d, d_{A}, d_{K}, \mu_{A}, \mu_{K}, \gamma, \alpha$ are parameters. $L_{0}, R_{0}, C_{0}, K_{0}$ and the function $g$ are given. The convective speeds $u_{k}$ and $u_{A}$ can be different in comparison with the speed growth $\mathrm{u}$. The goal of this article is to account for the change of size of branches. So we consider a simplified mathematical model where the diffusion is neglected and only the convection of one species is represented. Let us mention that the nutrient concentration evolution in the one branching case has been announced in [10], here we aim to simulate the transport of nutrient concentration for a multiple branching case. We deal with the transport equation in domains of different sizes. Due to the heterogeneous domain, the method of asymptotic partial domain decomposition MAPDD [9] could be used (which corresponds to consider only the first one basis function ). This method has been studied in [8] for an elliptic problem on a geometrical heterogeneous domains when the right hand side does not depend on the shrinking variable. A numerical finite element method is given for this problem in [7] and [6]. In this work we consider the transport equation in an heterogeneous domain with a general right hand side. Introducing a variational reduction type method, we show that the problem can be reduced to a $2 \mathrm{D}-1 \mathrm{D}$ problem. A numerical method coupling finite differences and asymptotic expansion is used for numerical simulations.

\section{Mathematical models}

\subsection{One branching case: the non reduced model}

Let us consider the transport equation in the following domain $Q$ representing trunk and one branch. 


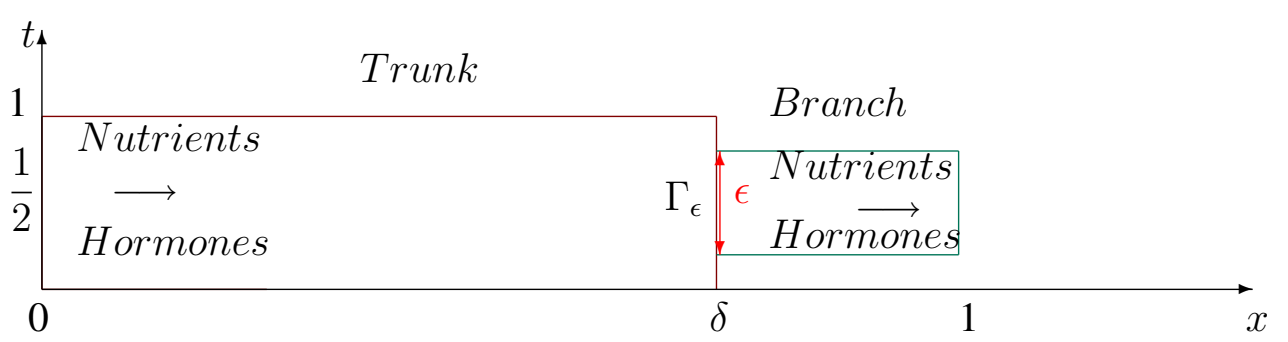

which is split into two sub-domains :

$$
Q=(0,1) \times(0, \delta) \cup\left(\frac{1}{2}-\epsilon, \frac{1}{2}+\epsilon\right) \times(\delta, 1)=Q_{1} \cup Q_{2} \quad ; \quad \Gamma_{\epsilon}=\left(\frac{1}{2}-\epsilon, \frac{1}{2}+\epsilon\right) \times\{\delta\}
$$

with a common boundary $\Gamma_{\epsilon}$. Let $f \in C^{0}(Q ; \mathbb{R})$ and $a \in C^{1}(Q ; \mathbb{R})$ be given, we define the transport velocity function $\beta=\left(\begin{array}{c}1 \\ a(t, x)\end{array}\right)$, and we assume $a$ to be bounded from below by a positive number. The Euclidean inner product of $\mathbb{R}^{2}$ is denoted by $(\cdot / \cdot)$, the outside normal to the domain $Q$ is denoted by $n$, and div stands for the divergence operator with respect to $(t, x)$ variables. Defining the incoming part of the boundary of the domain $Q$ by

$$
\partial Q_{-}=\{(t, x) \in \partial Q,(\beta / n)<0\}
$$

The functional space $H(\beta, Q)$ is defined by:

$$
H(\beta, Q)=\left\{\rho \in L^{2}(Q), \operatorname{div}(\beta \rho) \in L^{2}(Q),\left.\rho\right|_{\partial Q_{-}} \in L^{2}\left(\partial Q_{-},|(\beta / n)| d \sigma\right)\right\},
$$

where $L^{2}\left(\partial Q_{-},|(\beta / n)| d \sigma\right)$ stands for the space of $L^{2}$-functions defined on $\partial Q_{-}$with respect to the weighed measure of the boundary $\partial Q_{-}$.

The non reduced problem reads:

find $u \in H(\beta, Q)$ verifying the following system

$$
\begin{cases}(\beta / \nabla u)=\frac{\partial u}{\partial t}(t, x)+a(t, x) \frac{\partial u}{\partial x}(t, x)=f(t, x) & \text { in } Q \\ u=0 & \text { on } \partial Q_{-} .\end{cases}
$$

Taking into account of the hypotheses for transport velocity function and the right hand side function, an existence and uniqueness result for the solution $u$ to Problem (2.1) is given in [1]. It is well known that, when the finite difference methods are used for the approximation space, some numerical discrepancies appear when the thickness of the domain $Q_{2}$ is small and the time step is adapted only to the field $Q_{1}$ (see example [10] ). Therefore we are going to consider a reduced model in the next section to avoid these difficulties.

\subsection{One branching case: a variational reduced model}

From now on, the solution $u$ to Problem (2.1) is sought in different form in each sub-domain $Q_{i}$. This will lead to formulations of domain decomposition for Problem (2.1). Let $H^{1}(\delta, 1)$ denote the 
classical one dimensional Sobolev's space and let $M\left(\Gamma_{\epsilon}\right) \subset L^{2}\left(\Gamma_{\epsilon}\right)$ be a finite dimensional subset. For $i \in\{1,2\}$, the functions $u_{i}$ will represent in a certain sense the restrictions of the function $u$ to $Q_{i}$. Furthermore $u_{1} \in H\left(\beta, Q_{1}\right)$ and $u_{2} \in M\left(\Gamma_{\epsilon}\right) \otimes H^{1}(\delta, 1)$. We specify that the space $M\left(\Gamma_{\epsilon}\right)$ is built so that $M\left(\Gamma_{\epsilon}\right)=\operatorname{span}\left(\left(q_{i}\right)_{\{i=0,1, . . m\}}\right), m \in \mathbb{N}$, where we assume that the functions $\left(q_{i}\right)_{\{i=0,1, \ldots, m\}}$ satisfy:

- $\left(q_{j}\right)_{0 \leq j \leq m}$ are orthogonal with respect to the inner product of $L^{2}\left(\Gamma_{\epsilon}, a(., \delta) d t\right)$;

- $\left.\int_{\frac{1}{2}-\epsilon}^{\frac{1}{2}+\epsilon} q_{k}^{\prime}(t)\right) q_{j}(t) d t=0$ for $0 \leq k, j \leq m$ where $q_{k}^{\prime}$ denotes the derivative.

A reduced variational formulation for Problem (2.1) reads:

$$
\left\{\begin{array}{l}
\text { Find }\left(u_{1}, u_{2}\right) \in H\left(\beta, Q_{1}\right) \times M\left(\Gamma_{\epsilon}\right) \otimes H^{1}(\delta, 1) \text { verifying } \\
\int_{Q_{1}} \frac{\partial u_{1}}{\partial t}(t, x) \psi(t, x)+a(t, x) \frac{\partial u_{1}}{\partial x} \psi(t, x) d t d x=\int_{Q_{1}} f(t, x) \psi(t, x) d t d x, \quad \forall \psi \in L^{2}\left(Q_{1}\right) ; \\
\left.u_{1}\right|_{\partial Q_{1_{-}}}=0 ; \\
b\left(u_{1}-u_{2}, q\right)=\int_{\frac{1}{2}-\epsilon}^{\frac{1}{2}+\epsilon}\left(u_{1}(t, \delta)-u_{2}(t, \delta)\right) q(t) a(t, \delta) d t=0, \quad \forall q \in M\left(\Gamma_{\epsilon}\right) ; \\
\int_{Q_{2}} a(t, x) \frac{\partial u_{2}}{\partial x} q(t) \varphi(x) d t d x=\int_{Q_{2}} f(t, x) q(t) \varphi(x) d t d x, \quad \forall(\varphi, q) \in L^{2}(\delta, 1) \times M\left(\Gamma_{\epsilon}\right) .
\end{array}\right.
$$

The continuity condition on $\Gamma_{\epsilon}$ is expressed with the bilinear form $b(\cdot, \cdot)$ according to the trace results for functions belonging to $H\left(\beta, Q_{1}\right)$ (see [1]). The problem on the domain $Q_{2}$ is reduced to a differential equation, the initial condition at $x=\delta$ is obtained from the projection of the solution $u_{1}$ on each basis function $q_{j}$.

In the following lemma, when the function $a$ is time independent, a basis of subspace $M\left(\Gamma_{\epsilon}\right)$ is given [10].

Lemma 1. Let $m \in \mathbb{N}$, be fixed. Suppose a (., .) to be time independent, then assign to $q_{0}(t)=1$ and to $q_{j}(t)=\sin \left[j \pi\left(\frac{t-\frac{1}{2}}{\varepsilon}+1\right)\right]$ for all $1 \leq j \leq m$ and $t \in\left(\frac{1}{2}-\epsilon, \frac{1}{2}+\epsilon\right)$. The functions $\left(q_{j}\right)_{0 \leq j \leq m}$

- are orthogonal with respect to the scalar product of $L^{2}\left(\Gamma_{\epsilon}, a(., \delta) d t\right)$;

- and verify $\int_{\frac{1}{2}-\epsilon}^{\frac{1}{2}+\epsilon} q_{k}^{\prime}(t) q_{j}(t) d t=0$ for $0 \leq k, j \leq m$ where $q_{k}^{\prime}$ denotes the derivative function of $q_{k}$. 
Proof. It is easy to check the claimed properties with straightforward computations since the functions $q_{j}$ are orthogonal with respect to the scalar product of $L^{2}\left(\Gamma_{\epsilon}\right)$. It is to be noticed that the family $\left(q_{j}\right)_{0 \leq j \leq m}$ is dense in $L^{2}$ and $H_{0}^{1}$. $\square$ Unlike in [10], we are able in this work to deal the general case thanks to the following result.

Lemma 2. Let $m \in \mathbb{N}$, be fixed. Suppose a (., .) to be $C^{1}$ and bounded from below by a positive constant, then there exists a family of regular functions $\left(v_{j}\right)_{0 \leq j \leq m}$ for $t \in\left(\frac{1}{2}-\epsilon, \frac{1}{2}+\epsilon\right)$ which generates $M\left(\Gamma_{\epsilon}\right)$. In addition functions $\left(v_{j}\right)_{0 \leq j \leq m}$, for $t \in\left(\frac{1}{2}-\epsilon, \frac{1}{2}+\epsilon\right)$,

- are orthogonal with respect to the scalar product of $L^{2}\left(\Gamma_{\epsilon}, a(., \delta) d t\right)$;

- and verify $\int_{\frac{1}{2}-\epsilon}^{\frac{1}{2}+\epsilon} v_{k}^{\prime}(t) v_{j}(t) d t=0$ for $0 \leq k, j \leq m$ where $v_{k}^{\prime}$ denotes the derivative function of $v_{k}$.

Proof. Let $\left(\tilde{q}_{j}\right)_{0 \leq j \leq m}$ be the normalized basis derived from the $L^{2}$-orthogonal basis $\left(q_{j}\right)_{0 \leq j \leq m}$. Since the function $a$ is bounded from below by a positive constant, the bilinear form $\int_{\frac{1}{2}-\epsilon}^{\frac{1}{2}+\epsilon} \cdots d t$ defines an $L^{2}$-inner product. Let us denote by $M$ its matrix with respect to the basis $\left(\tilde{q}_{j}\right)_{0 \leq j \leq m}$. The matrix $M$ is symmetric positive definite, thus the spectral theorem claims there exists an orthogonal basis with respect to $L^{2}$-inner product denoted by $\left(v_{j}\right)_{0 \leq j \leq m}$, which diagonalizes the matrix $M$. The expression of the bilinear form in the basis $\left(v_{j}\right)_{0 \leq j \leq m}$ is diagonal, thus the vectors $v_{j}$ are orthogonal to each others with respect to the bilinear form. Since the $v_{j}$ 's functions are linear combinations of $\tilde{q}_{i}$ 's functions, the second items of Lemma 1 still holds true for the $v_{j}$ 's functions. Lemma 2 is proved.

Due to the orthogonal basis, we just have to solve ordinary differential equations with respect to the $x$ variable in the domains $Q_{2}$ that which prevent to difficulties with the sizing of the time step for the numerical approximation. In the case where only the first basis functions are kept we have:

$$
\begin{cases}\text { Find } u_{1} \in H\left(\beta, Q_{1}\right) \text { and a function } & u_{20} \in H^{1}(\delta, 1) \text { such that } \\ \left(\beta, \nabla u_{1}\right)_{2}(t, x)=f(t, x) & , \forall(t, x) \in Q_{1} \\ \frac{d\left(u_{20}(x)\right)}{d x}=\frac{\int_{\frac{1}{2}-\epsilon}^{\frac{1}{2}+\epsilon} f(t, x) d t}{\int_{\frac{1}{2}-\epsilon}^{\frac{1}{2}+\epsilon} a(t, x) d t} & , \forall \delta \leq x \leq 1 \\ \int_{\frac{1}{2}-\epsilon}^{\frac{1}{2}+\epsilon} a(t, \delta) u_{1}(t, \delta) d t & , x=\delta \\ \int_{\frac{1}{2}-\epsilon}^{\frac{1}{2}+\epsilon} a(t, \delta) d t & \end{cases}
$$


Let us remark that when $\epsilon$ goes to zero the zero order approximation of Problem (2.2) is given by

$$
\left\{\begin{array}{lc}
\text { Find } u_{1} \in H\left(\beta, Q_{1}\right) & \text { and } u_{20} \in H^{1}(\delta, 1) \text { such that } \\
\left(\beta, \nabla u_{1}\right)_{2}=f(t, x), & (t, x) \in Q_{1} \\
\frac{d\left(u_{20}(x)\right)}{d x}=\frac{f\left(\frac{1}{2}, x\right)}{a\left(\frac{1}{2}, x\right)} & \delta \leq x \leq 1 \\
u_{20}(\delta)=u_{1}\left(\frac{1}{2}, \delta\right) . &
\end{array}\right.
$$

Remark 1 If the function $\tilde{u}$ is defined by $\tilde{u}=\left\{\begin{array}{c}u_{1} \text { in } Q_{1} ; \\ u_{2} \text { in } Q_{2}\end{array}\right.$, then one can prove that $\tilde{u} \in H(\beta, Q)$. The discrepancy between $u$ solution to Problem (2.1) and $\tilde{u}$ is proportional to the discrepancy between $L^{2}\left(\Gamma_{\epsilon}\right)$ and $M\left(\Gamma_{\epsilon}\right)$, that is to say proportional to $\frac{1}{m}$ if the functions are sufficiently regular, and if the function $f$ is symmetric.

Now, let us come to the numerical approximation of $\left(u_{1}, u_{2}\right)$ solution to the decomposed problem (2.2). To supplement the results outlined in [10], We will give in that follows the construction of the algorithm necessary to the numerical simulations. An upwind finite differences method is used for $u_{1}$ in $Q_{1}$ and for $u_{2}$ in $Q_{2}$, and to avoid technicalities, only the $q_{0}$ function of the basis of $M\left(\Gamma_{\epsilon}\right)$ is used. Thus, the function $u_{2}(t, x)$ is approximated by $q_{0}(t) u_{20}(x)$ for all $(t, x) \in Q_{2}$. Let $N, P \in \mathbb{N}$ be two fixed integers, the time and space steps are defined by:

$$
\Delta t=\frac{1}{N} ; \quad \Delta x=\frac{1}{J} ; \quad \delta=J \Delta x .
$$

Introduce the family of points $\left\{t^{n}, x_{j}\right\}$ defined by:

$$
t^{n}=n \Delta t ; \quad x_{j}=j \Delta x \text { for } 0 \leq n \leq N ; \quad 0 \leq j \leq P .
$$

Since, the function $a$ is non negative, the incoming part of the boundary will be constituted of $\{0\} \times(0, \delta) \cup(0,1) \times\{0\}$. We denote by $A$ the $J \times J$ matrix defined by:

$$
A=I+\frac{\Delta t}{\Delta x}\left(\begin{array}{cccc}
a\left(t^{n+1}, x_{1}\right) & 0 & \cdot & 0 \\
-a\left(t^{n+1}, x_{2}\right) & a\left(t^{n+1}, x_{2}\right) & 0 & 0 \\
0 & \cdot & \cdot & \cdot \\
\cdot & \cdot & \cdot & 0 \\
0 & 0 & -a\left(t^{n+1}, x_{J}\right) & a\left(t^{n+1}, x_{J}\right)
\end{array}\right)
$$

and by $U_{1}^{n+1} ; F^{n+1}$ the $\mathbb{R}^{J}$ vectors which respectively represent a sampling of the function $u_{1}$ and of the right hand side, at time $t^{n+1}$ and for every points $\left\{x_{j}\right\}_{j=1}^{j=J}$ :

$$
F^{n+1}=\left(\begin{array}{c}
f\left(t^{n+1}, x_{1}\right) \\
f\left(t^{n+1}, x_{2}\right) \\
\cdot \\
\cdot \\
f\left(t^{n+1}, x_{J}\right)
\end{array}\right)
$$


If the linear Lagrange's time interpolation operator is denoted by $\pi_{N}$, then we have the following result.

Proposition 3. For $U_{1}^{0}=0$

$$
\left\{\begin{array}{l}
U_{1}^{n+1} \in \mathbb{R}^{J} \text { and } ; u_{20}\left(x_{i}\right) \text { are solutions to: } \\
A U_{1}^{n+1}=F^{n+1} \quad, 0 \leq n \leq N-1 \\
u_{20}(J)=\frac{\int_{\frac{1}{2}-\epsilon}^{\frac{1}{2}+\epsilon} a(t, \delta) \pi_{N} U_{1 J} d t}{\int_{\frac{1}{2}-\epsilon}^{\frac{1}{2}+\epsilon} a(t, \delta) d t} \quad, \\
u_{20}\left(x_{i+1}\right)=u_{20}\left(x_{i}\right)+\Delta x \frac{\int_{\frac{1}{2}-\epsilon}^{\frac{1}{2}+\epsilon} f\left(t, x_{i+1}\right) d t}{\int_{\frac{1}{2}-\epsilon}^{\frac{1}{2}+\epsilon} a\left(t, x_{i+1}\right) d t} \quad, J \leq i \leq P-1 .
\end{array}\right.
$$

The vectors $U_{1} \in \mathbb{R}^{J} ; u_{20}(\cdot) \in \mathbb{R}^{N-J+1}$ represent a numerical approximation to Problem (2.2).

Proof. We look for a solution to problem (2.2) such that $u_{2}$ is approximated by $u_{20} q_{0}$ where $q_{0}$ is defined in Lemma 1. By using the orthogonality results given in lemma 2 the equation (2.2) becomes for all $\varphi \in L^{2}(\delta, 1)$

$$
\int_{\delta}^{1}\left(\int_{\frac{1}{2}-\epsilon}^{\frac{1}{2}+\epsilon} a(t, x) \frac{\left(u_{20}(x)\right)}{d x} q_{0}(t) d t-\int_{\frac{1}{2}-\epsilon}^{\frac{1}{2}+\epsilon} f(t, x) q_{0}(t) d t\right) \varphi(x) d x=0 .
$$

Since the $C^{\infty}$ functions compactly supported in $(\delta, 1)$, verify $\mathcal{D}(\delta, 1) \subset L^{2}(\delta, 1)$, the following equality holds in $\mathcal{D}^{\prime}(\delta, 1)$

$$
\int_{\frac{1}{2}-\epsilon}^{\frac{1}{2}+\epsilon} a(t, x) \frac{d\left(u_{20}(x)\right)}{d x} d t=\int_{\frac{1}{2}-\epsilon}^{\frac{1}{2}+\epsilon} f(t, x) d t .
$$

The relation $b_{1}\left(u_{1}-u_{2 M}, q_{0}\right)=0$ gives $\int_{\frac{1}{2}-\epsilon}^{\frac{1}{2}+\epsilon}\left(u_{1}(t, \delta)-u_{20}(\delta)\right) q_{0}(t) a(t, \delta) d t=0$ then

$$
\int_{\frac{1}{2}-\epsilon}^{\frac{1}{2}+\epsilon} a(t, \delta) u_{1}(t, \delta) d t=u_{20}(\delta) \int_{\frac{1}{2}-\epsilon}^{\frac{1}{2}+\epsilon} a(t, \delta) d t .
$$

Let us end the proof by proving that $U_{1}^{n+1}$ is computable. It is well known that the matrix $A$ is a M-matrix, thus invertible with $A^{-1}$ a positive matrix. 


\subsection{Two branching case}

Let us consider the Transport equation in the domain $Q$ defined as follows:

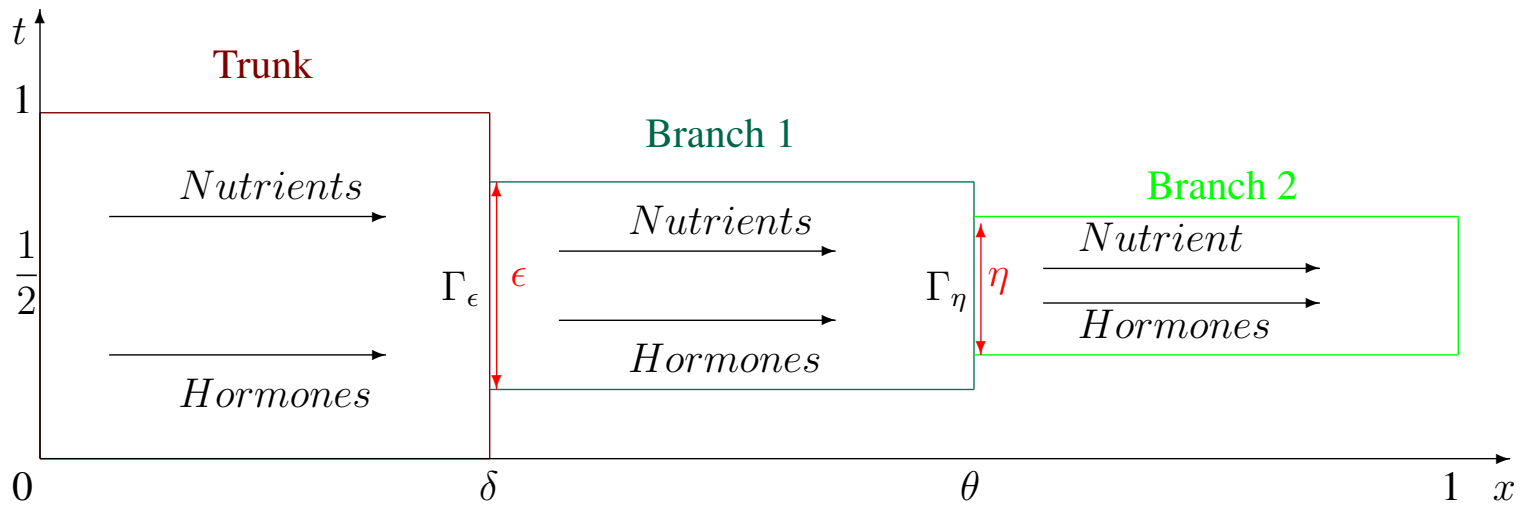

An heterogeneous domain $\mathrm{Q}$ representing trunk and two branches of a plant.

$Q=(0,1) \times(0, \delta) \cup\left(\frac{1}{2}-\epsilon, \frac{1}{2}+\epsilon\right) \times(\delta, \theta) \cup\left(\frac{1}{2}-\eta, \frac{1}{2}+\eta\right) \times(\theta, 1)=Q_{1} \cup Q_{2} \cup Q_{3}$

$\Gamma_{\epsilon}=\left(\frac{1}{2}-\epsilon, \frac{1}{2}+\epsilon\right) \times\{\delta\}$ and $\Gamma_{\eta}=\left(\frac{1}{2}-\eta, \frac{1}{2}+\eta\right) \times\{\theta\}$

We assume that the transport velocity satisfies the same hypothesis as in the previous subsection, and we look for $u \in H(\beta, Q)$ verifying:

$$
\left\{\begin{array}{l}
(\beta / \nabla u)=\frac{\partial u}{\partial t}(t, x)+a(t, x) \frac{\partial u}{\partial x}(t, x)=f(t, x) \\
u=0 \text { on } \partial Q_{-}
\end{array}\right.
$$

An existence and uniqueness result of a solution $u$ to the problem (2.7) is given in [1] for example. We denote by $u_{i}$ the restriction of $u$ on $Q_{i}$ where $i=1,2,3$. Then the decomposed problem associated to the problem (2.7) reads: Find $\left(u_{1}, u_{2}, u_{3}\right) \in H\left(\beta, Q_{1}\right) \times H\left(\beta, Q_{2}\right) \times H\left(\beta, Q_{3}\right)$ verifying

$$
\left\{\begin{array}{l}
\int_{Q_{1}} \frac{\partial u_{1}}{\partial t}(t, x) \psi(t, x)+a(t, x) \frac{\partial u_{1}}{\partial x} \psi(t, x) d t d x=\int_{Q_{1}} f(t, x) \psi(t, x) d t d x, \quad \forall \psi \in L^{2}\left(Q_{1}\right) ; \\
\left.u_{1}\right|_{\partial Q_{1}}=0 ; \\
\int_{Q_{2}} \frac{\partial u_{2}}{\partial t}(t, x) \varphi(t, x)+a(t, x) \frac{\partial u_{2}}{\partial x} \varphi(t, x) d t d x=\int_{Q_{2}} f(t, x) \varphi(t, x) d t d x, \quad \varphi \in L^{2}\left(Q_{2}\right) ; \\
b_{1}\left(u_{1}-u_{2}, q\right)=\int_{\frac{1}{2}-\epsilon}^{\frac{1}{2}+\epsilon}\left(u_{1}(t, \delta)-u_{2}(t, \delta)\right) q(t) a(t, \delta) d t=0, \quad \forall q \in L^{2}\left(\Gamma_{\epsilon}\right) \\
\int_{Q_{3}} \frac{\partial u_{3}}{\partial t}(t, x) \xi(t, x)+a(t, x) \frac{\partial u_{3}}{\partial x} \xi(t, x) d t d x=\int_{Q_{3}} f(t, x) \xi(t, x) d t d x, \quad \forall \xi \in L^{2}\left(Q_{3}\right) ; \\
b_{2}\left(u_{2 M}-u_{3}, p\right)=\int_{\frac{1}{2}-\eta}^{\frac{1}{2}+\eta}\left(u_{2}(t, \theta)-u_{3}(t, \theta)\right) p(t) a(t, \theta) d t=0, \quad \forall p \in L^{2}\left(\Gamma_{\eta}\right) .
\end{array}\right.
$$


The interface conditions on the boundaries $\Gamma_{\epsilon}$ and $\Gamma_{\eta}$ are given with a weighted $L^{2}$ inner product according to the trace results for the spaces $H\left(\beta, Q_{i}\right)$ for $1 \leq i \leq 3$.

\subsubsection{A variational reduced method}

Let $\left(m, m^{\prime}\right) \in \mathbb{N}^{2}$. We choose $q_{0}(t)=1, q_{j}(t)=\sin \left(j \pi\left(\frac{t-\frac{1}{2}}{\epsilon}+1\right)\right) \quad \forall 1 \leq j \leq m, p_{0}(t)=1$ and $p_{j}(t)=\sin \left(j \pi\left(\frac{t-\frac{1}{2}}{\eta}+1\right)\right) \forall 1 \leq j \leq m^{\prime}$. The functions introduced bellow verify the same properties as in lemma 1.Define $M\left(\Gamma_{\epsilon}\right)=\operatorname{span}\left(\left\{q_{i}\right\}_{i=0}^{i=m}\right)$ and $M\left(\Gamma_{\eta}\right)=\operatorname{span}\left(\left\{p_{j}\right\}_{j=0}^{j=m^{\prime}}\right)$. Then as in the previous subsection one can prouve the following result:

Lemma 4. Let $\left(m, m^{\prime}\right) \in \mathbb{N}^{2}$, be fixed. Suppose a (.,.) to be $C^{1}$ and bounded from below by a positive constant, then there exist families of regular functions $\left(v_{j}\right)_{0 \leq j \leq m} ;\left(w_{k}\right)_{0 \leq k \leq m^{\prime}}$ for $t \in\left(\frac{1}{2}-\epsilon, \frac{1}{2}+\epsilon\right)$ which respectively generate $M\left(\Gamma_{\epsilon}\right) ; M\left(\Gamma_{\eta}\right)$ and

- which are respectively orthogonal with respect to the scalar product of $L^{2}\left(\Gamma_{\epsilon}, a(., \delta) d t\right)$; $L^{2}\left(\Gamma_{\eta}, a(., \theta) d t\right)$

- and which respectively verify $\int_{\frac{1}{2}-\epsilon}^{\frac{1}{2}+\epsilon} v_{k}^{\prime}(t) v_{j}(t) d t=0$ for $0 \leq k, j \leq m$; $\int_{\frac{1}{2}-\eta}^{\frac{1}{2}+\eta} w_{k}^{\prime}(t) w_{j}(t) d t=0$ for $0 \leq k, j \leq m^{\prime}$ where $v_{k}^{\prime}$ and $w_{k}^{\prime}$ denote the derivatives.

In order to introduce the reduced variational formulation, let us define the following product spaces $H\left(\beta, Q_{1}\right) \times M\left(\Gamma_{\epsilon}\right) \otimes H^{1}(\delta, \theta) \times M\left(\Gamma_{\eta}\right) \otimes H^{1}(\theta, 1)$. In the same way as for the one branching case the problem (2.7) can be reduced to the following system:

$\left\{\begin{array}{l}\text { Find }\left(u_{1}, u_{2}, u_{3}\right) \in H\left(\beta, Q_{1}\right) \times M\left(\Gamma_{\epsilon}\right) \otimes H^{1}(\delta, \theta) \times M\left(\Gamma_{\eta}\right) \otimes H^{1}(\theta, 1) \quad \text { verifying } \\ \int_{Q_{1}} \frac{\partial u_{1}}{\partial t}(t, x) \psi(t, x)+a(t, x) \frac{\partial u_{1}}{\partial x}(t, x) \psi(t, x) d t d x=\int_{Q_{1}} f(t, x) \psi(t, x) d t d x, \quad \forall \psi \in L^{2}\left(Q_{1}\right) ; \\ \left.u_{1}\right|_{\partial Q_{1_{-}}}=0 ; \\ b\left(u_{1}-u_{2}, q\right)=\int_{\frac{1}{2}-\epsilon}^{\frac{1}{2}+\epsilon}\left(u_{1}(t, \delta)-u_{2}(t, \delta)\right) q(t) a(t, \delta) d t=0, \quad \forall q \in M\left(\Gamma_{\epsilon}\right) ; \\ \int_{Q_{2}} a(t, x) \frac{\partial u_{2}}{\partial x} q(t)(t, x) \varphi(x) d t d x=\int_{Q_{2}} f(t, x) q(t) \varphi(x) d t d x, \quad \forall(\varphi, q) \in L^{2}(\delta, \theta) \times M\left(\Gamma_{\epsilon}\right) ; \\ b\left(u_{2}-u_{3}, p\right)=\int_{\frac{1}{2}-\eta}^{\frac{1}{2}+\eta}\left(u_{2}(t, \theta)-u_{3}(t, \theta)\right) p(t) a(t, \delta) d t=0, \quad \forall p \in M\left(\Gamma_{\eta}\right) ; \\ \int_{Q_{3}} a(t, x) \frac{\partial u_{2}}{\partial x}(t, x) p(t) \xi(x) d t d x=\int_{Q_{3}} f(t, x) p(t) \xi(x) d t d x, \quad \forall(\xi, p) \in L^{2}(\theta, 1) \times M\left(\Gamma_{\eta}\right) .\end{array}\right.$ 
Due to the orthogonal basis we just have to solve ordinary differential equations over the variable x in the field $Q_{2}$ and $Q_{3}$ which overcomes the difficulties related to the size of time step for the numerical approximation. In the case where only the first basis functions are kept we have:

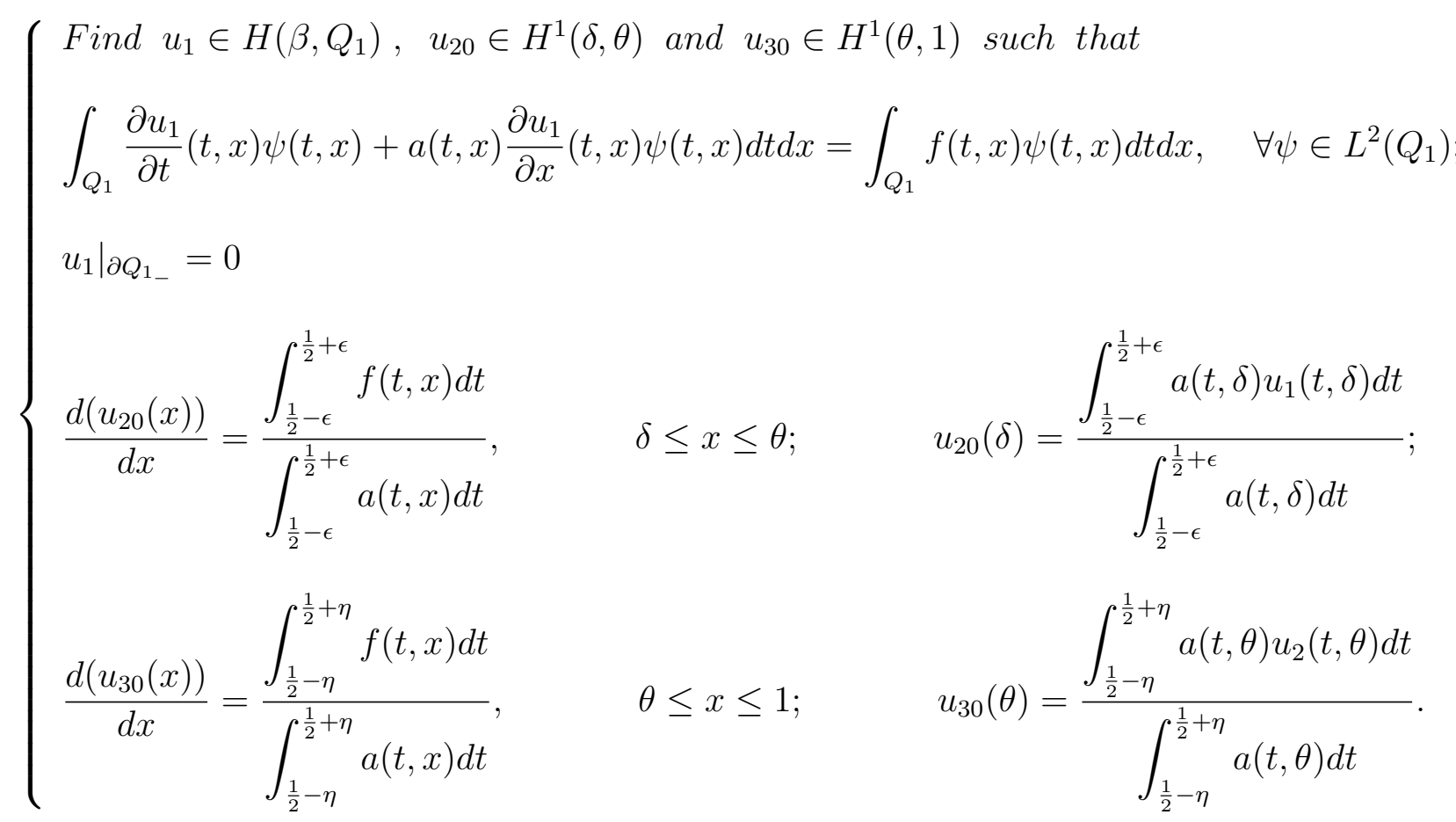

When $\epsilon$ and $\eta$ go to zero, we obtain the following zero order approximation of the problem (2.7)

$$
\left\{\begin{array}{l}
\text { Find } u_{1} \in H\left(\beta, Q_{1}\right), u_{20} \in H^{1}(\delta, \theta) \text { and } u_{30} \in H^{1}(\theta, 1) \text { such that } \\
\int_{Q_{1}} \frac{\partial u_{1}}{\partial t}(t, x) \psi(t, x)+a(t, x) \frac{\partial u_{1}}{\partial x}(t, x) \psi(t, x) d t d x=\int_{Q_{1}} f(t, x) \psi(t, x) d t d x, \quad \forall \psi \in L^{2}\left(Q_{1}\right) \\
\left.u_{1}\right|_{\partial Q_{1_{-}}}=0 \\
\frac{d\left(u_{20}(x)\right)}{d x}=\frac{f\left(\frac{1}{2}, x\right)}{a\left(\frac{1}{2}, x\right)}, \quad \delta \leq x \leq \theta ; \quad u_{20}(\delta)=u_{1}\left(\frac{1}{2}, \delta\right) \\
\frac{d\left(u_{30}(x)\right)}{d x}=\frac{f\left(\frac{1}{2}, x\right)}{a\left(\frac{1}{2}, x\right)}, \quad \theta \leq x \leq 1 ; \quad u_{30}(\theta)=u_{2}\left(\frac{1}{2}, \theta\right)
\end{array}\right.
$$

Let us mention that a part of the results given in this subsection have been presented in [2] and [3]. In this work, due to the lemma 4, we are able to deal with the general case and we focus on the numerical treatment and simulation of the equations of systems (2.9) and (2.10). 


\section{Numerical simulations}

In this section some numerical simulations are presented. We take for the velocity function $a(t, x)=50 x+\exp (x)$ and for the right hand side function $f(t, x)=x+x \exp \left(\frac{t}{2}\right)$.

The upwind scheme presented in Equation (2.6)is used for the domain $Q_{1}$. To solve the ordinary differential equations in $Q_{2}$ for (2.3) and for (2.4) in one branching case, respectively in $Q_{2}$ and in $Q_{3}$ for (2.9) and for (2.10) in multi-branching case we need to compute numerically integrals on $\Gamma_{\epsilon}$ or on $\Gamma_{\eta}$. We used for thats a quadrature Gauss's scheme [5]. For the ordinary differential equations a forward Euler's scheme have been implemented [5].

In Figure 1, we present numerical simulations for the one branching case. We take $\epsilon=0.25$ and we compare the finite differences method and the variational reduction with one sinus basis function in the case where time and space steps equal to $510^{-3}$ and $\epsilon=8.310^{-3}$.

In Figure 2, the case of two branches is considered. The first row represents a finite differences method for time and space steps equal to $110^{-2}$. We take $\epsilon=0.25$ in the first and second column respectively for $\eta=0.1$ and $\eta=0.01$. In the third column $\epsilon$ and $\eta$ are respectively 0.025 and 0.01 . Some numerical discrepancies appear for the latter cases. The second row represents the zero order model in the first column and the variational reduction method with one sinus basis function in the second and third column respectively for $\epsilon=0.25 ; \eta=0.1$ and for $\epsilon=0.025 ; \eta=0.01$. We remark that the discrepancies have disapeared in the last cases.

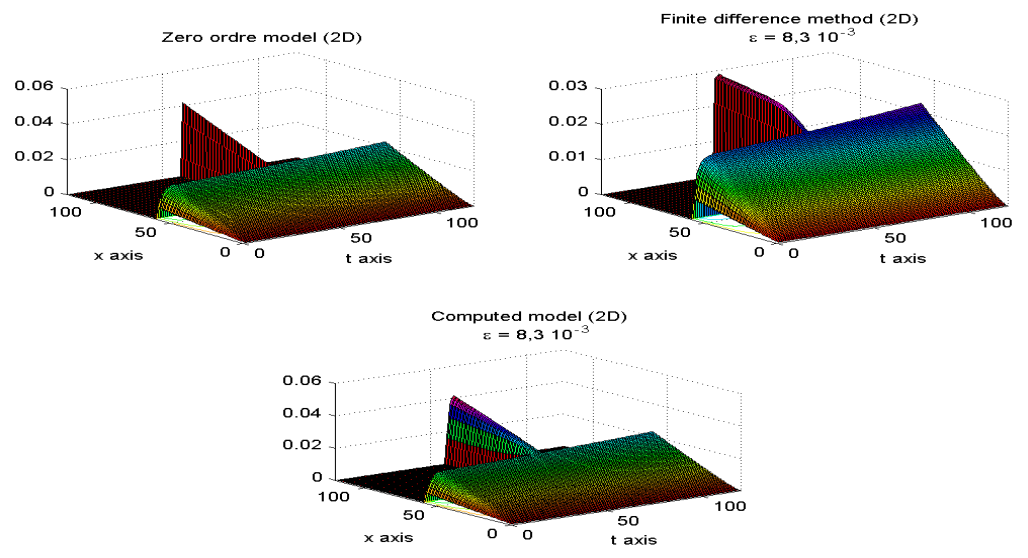

Figure 1: One branching case 

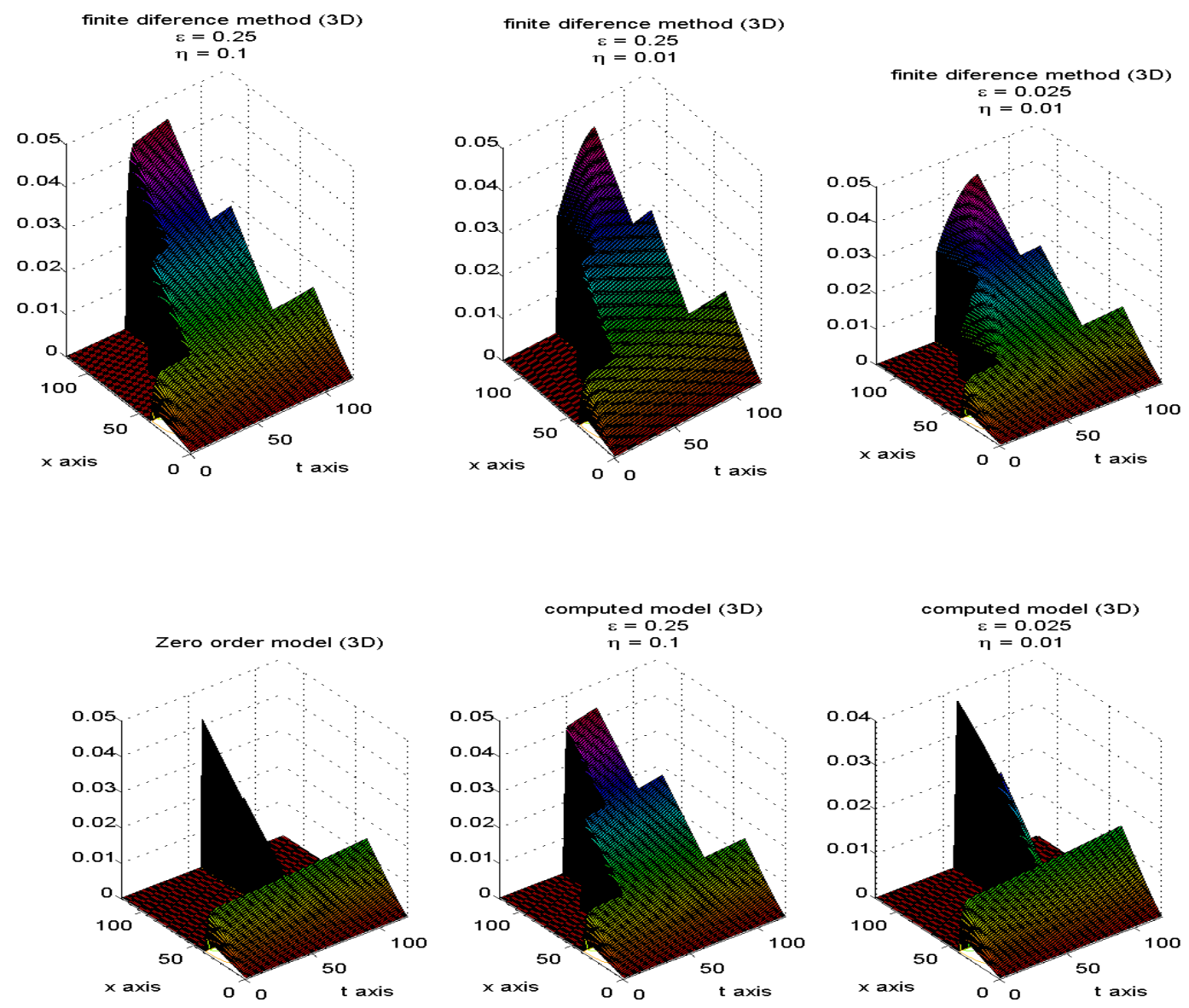

Figure 2: Two branching case

\section{Conclusion and Perspectives}

The numerical simulations show that the variational reduction method allows to get ride of the troubles due to the heterogeneous domains (or the necessity to take different sizes of time steps according to the size of the domain). If the numerical approximation for the concentration evolution in case of the zero order method is not sufficiently accurate compare to the classical finite differences method, we observe that the variational reduction method allows to improve the numerical results without changing the size of the time step. Heterogeneous domains can be handle with the presented method, without using very small time steps. The presented method should be extended to three dimensional cases by using cylindrical coordinates, which is a work in progress. 


\section{References}

[1] O. Besson, J. Pousin. Solution for Linear Conservation Laws with velocity in $L^{\infty}$. Archive for Rational Mechanics and Analysis, 2007.

[2] S. Boujena, A. Chiboub, J. Pousin. Variational reduction for the transport equation in a multiple branching plant growth model. Congrès International JANO 9, $9^{\text {emes }}$ journée d'Analyse Numérique et d'Optimisation. Mohammedia, Maroc, 2008.

[3] S. Boujena, A. Chiboub, J. Pousin. Variational reduction for the transport equation in a multiple branching plant growth model. Mathematical Modelling of Natural Phenomena, 5 (Supplement 2010), No. 7, 11-15.

[4] N. Bessonov, V. Volpert. Dynamic models of plant growth, Mathematics and mathematical modeling. Publibook, Paris, 2007.

[5] M. Crouzeix, L. Mignot. Analyse numérique des équations différentielles. Masson, Paris, 1996.

[6] F. Fontvieille, Décomposition asymptôtique et éléments finis. Thèse de doctorat, université Claude Bernard- Lyon I, 2004.

[7] F. Fontvieille, G. Panasenko, J. Pousin. FEM implementation for the asymptotic partial decomposition. Applicable Analysis, 86 (2007), No. 5 , 519-536.

[8] G.P. Panasenko. Multi-scale Modelling for structures and composites. Springer Verlag, 2005.

[9] G.P. Panasenko. Method of asymptotic partial decomposition of domain. Math. Models and Methods in Appl. Sci., 1 (1998), No. 8, 139-156.

[10] M. Picq, J. Pousin. Variational reduction for the transport equation and plants growth. In Proccedings of the Conference Modelling of the Heterogeneous Materials with Applications in Constructions and Biological Engineering. Czech Technical University Prague, 2007. 